\title{
A Certain Subclass of Analytic Functions with Bounded Positive Real Part
}

\author{
Young Jae Sim, Oh Sang Kwon \\ Department of Mathematics, Kyungsung University, Busan, South Korea \\ Email: yjsim@ks.ac.kr, oskwon@ks.ac.kr
}

Received March 6, 2013; revised April 11, 2013; accepted May 10, 2013

Copyright (C) 2013 Young Jae Sim, Oh Sang Kwon. This is an open access article distributed under the Creative Commons Attribution License, which permits unrestricted use, distribution, and reproduction in any medium, provided the original work is properly cited.

\section{ABSTRACT}

For real numbers $\alpha$ and $\beta$ such that $0 \leq \alpha<1<\beta$, we denote by $T(\alpha, \beta)$ the class of normalized analytic functions which satisfy $\alpha<\operatorname{Re}\left\{\sqrt{f^{\prime}(z)}\right\}<\beta \quad(z \in \mathbb{U})$, where $\mathbb{U}$ denotes the open unit disk. We find some relationships involving functions in the class $T(\alpha, \beta)$. And we estimate the bounds of coefficients and solve Fekete-Szegö problem for functions in this class. Furthermore, we investigate the bounds of initial coefficients of inverse functions or bi-univalent functions.

Keywords: Functions of Bounded Positive Real Part; Fekete-Szegö Problem; Inverse Functions; Bi-Univalent Functions

\section{Introduction}

Let $A$ denote the class of analytic functions in the unit disk $\mathbb{U}=\{z \in \mathbb{C}:|z|<1\}$ which is normalized by

$f(0)=0$ and $f^{\prime}(0)=1$. Also let $S$ denote the subclass of $A$ which is composed of functions which are univalent in $\mathbb{U}$.

We say that $f$ is subordinate to $F$ in $\mathbb{U}$, written as $f \prec F \quad(z \in \mathbb{U})$, if and only if $f(z)=F(w(z))$ for some Schwarz function $w(z)$ such that $w(0)=0$ and $|w(z)|<1 \quad(z \in \mathbb{U})$. If $F$ is univalent in $\mathbb{U}$, then the subordination $f \prec F$ is equivalent to $f(0)=F(0)$ and $f(\mathbb{U}) \subset F(\mathbb{U})$.

Definition 1.1. Let $\alpha$ and $\beta$ be real numbers such that $0 \leq \alpha<1<\beta$. The function $f \in A$ belongs to the class $T(\alpha, \beta)$ if $f$ satisfies the following inequality:

$$
\alpha<\operatorname{Re}\left\{\sqrt{f^{\prime}(z)}\right\}<\beta \quad(z \in \mathbb{U}) .
$$

We remark that, for given real numbers $\alpha$ and $\beta$ $(0 \leq \alpha<1<\beta), f \in T(\alpha, \beta)$ if and only if $f$ satisfies each of the following two subordination relationships:

$$
\sqrt{f^{\prime}(z)} \prec \frac{1+(1-2 \alpha) z}{1-z} \quad(z \in \mathbb{U})
$$

and

$$
\sqrt{f^{\prime}(z)} \prec \frac{1+(1-2 \beta) z}{1-z} \quad(z \in \mathbb{U}) .
$$

Now, we define an analytic function $p: \mathbb{U} \rightarrow \mathbb{C}$ by

$$
p(z)=1+\frac{\beta-\alpha}{\pi} \mathrm{i} \log \left(\frac{1-\mathrm{e}^{2 \pi \mathrm{i} \frac{1-\alpha}{\beta-\alpha}} \mathrm{z}}{1-\mathrm{z}}\right) .
$$

The above function $p$ was introduced by Kuroki and Owa [1] and they proved $p$ maps $\mathbb{U}$ onto a convex domain $\Lambda=\{w: \alpha<\operatorname{Re}\{w\}<\beta\}$, conformally. Using this fact and the definition of subordination, we can obtain the following Lemma, directly.

Lemma 1.1. Let $f(z) \in A$ and $0 \leq \alpha<1<\beta$. Then $f \in T(\alpha, \beta)$ if and only if

$$
\sqrt{f^{\prime}(z)} \prec 1+\frac{\beta-\alpha}{\pi} \mathrm{i} \log \left(\frac{1-\mathrm{e}^{2 \pi \mathrm{i} \frac{1-\alpha}{\beta-\alpha}} \mathrm{z}}{1-z}\right)
$$

in $\mathbb{U}$.

And we note that the function $p$, defined by (1), has the form $p(z)=1+\sum_{n=1}^{\infty} B_{n} z^{n}$, where

$$
B_{n}=\frac{\beta-\alpha}{n \pi} \mathrm{i}\left(1-\mathrm{e}^{2 n \pi i \frac{1-\alpha}{\beta-\alpha}}\right) \quad(n \in \mathbb{N}) .
$$


For given real numbers $\alpha$ and $\beta$ such that $0 \leq \alpha<1<\beta$, we denote $T_{\sigma}(\alpha, \beta)$ the class of biunivalent functions consisting the functions in $A$ such that $f \in T(\alpha, \beta)$ and $f^{-1} \in T(\alpha, \beta)$, where $f^{-1}$ is the inverse function of $f$.

In our present investigation, we first find some relationships for functions in bounded positive class $T(\alpha, \beta)$. And we solve several coefficient problems including Fekete-Szegö problems for functions in the class. Furthermore, we estimate the bounds of initial coefficients of inverse functions and bi-univalent functions. For the coefficient bounds of functions in special subclasses of $S$, the readers may be referred to the works [2-4].

\section{Relations Involving Bounds on the Real Parts}

In this section, we shall find some relations involving the functions in $T(\alpha, \beta)$. And the following Lemma will be needed in finding the relations.

Lemma 2.1 (see Miller and Mocanu [5]) Let $\Xi$ be a set in the complex plane $\mathbb{C}$ and let $b$ be a complex number such that $\operatorname{Re}\{b\}>0$. Suppose that a function $\psi: \mathbb{C}^{2} \times \mathbb{U} \rightarrow \mathbb{C}$ satisfies the condition

$$
\psi(\mathrm{i} \rho, \sigma ; z) \notin \Xi
$$

for all real $\rho, \sigma \leq-|b-\mathrm{i} \rho|^{2} /(2 \operatorname{Re}\{b\})$ and all $z \in \mathbb{U}$. If the function $p(z)$ defined by

$$
\begin{gathered}
p(z)=b+b_{1} z+b_{2} z^{2}+\cdots \text { is analytic in } \mathbb{U} \text { and if } \\
\psi\left(p(z), z p^{\prime}(z)\right) \in \Xi,
\end{gathered}
$$

then $\operatorname{Re}\{p(z)\}>0$ in $\mathbb{U}$.

Theorem 2.2. Let $f \in A, 1 / 2 \leq \alpha<1$ and

$$
\operatorname{Re}\left\{\sqrt{f^{\prime}(z)}\right\}>\alpha \quad(z \in \mathbb{U}) .
$$

Then

$$
\operatorname{Re}\left\{\frac{f(z)}{z}\right\}>\frac{2}{3} \alpha^{2}+\frac{1}{3} \quad(z \in \mathbb{U}) .
$$

Proof. We put

$$
\gamma=\frac{2}{3} \alpha^{2}+\frac{1}{3}
$$

and let

$$
p(z)=\frac{1}{1-\gamma}\left(\frac{f(z)}{z}-\gamma\right) .
$$

Then $p$ is analytic in $\mathbb{U}$ and $p(0)=1$. And

$$
\begin{aligned}
\sqrt{f^{\prime}(z)} & =\sqrt{(1-\gamma) p(z)+(1-\gamma) z p^{\prime}(z)+\gamma} \\
& =\psi\left(p(z), z p^{\prime}(z)\right),
\end{aligned}
$$

where

$$
\psi(r, s)=\sqrt{(1-\gamma) r+(1-\gamma) s+\gamma} .
$$

Using (4), we have

$\left\{\psi\left(p(z), z p^{\prime}(z)\right): z \in \mathbb{U}\right\} \subset\{w \in \mathbb{C}: \operatorname{Re}\{w\}>\alpha\}:=\Omega_{\alpha}$.

Now, let $\rho, \sigma \in \mathbb{R}$ with $\sigma \leq-\left(1+\rho^{2}\right) / 2$. And we shall find the maximum value of $\operatorname{Re}\{\psi(\mathrm{i} \rho, \sigma)\}$. Now, we put

$$
\psi(\mathrm{i} \rho, \sigma)=\sqrt{(1-\gamma) \mathrm{i} \rho+(1-\gamma) \sigma+\gamma}:=u+\mathrm{i} v,
$$

where $u$ and $v$ are real numbers. Then

$$
u^{2}-v^{2}=(1-\gamma) \sigma+\gamma
$$

and

$$
2 u v=(1-\gamma) \rho .
$$

Hence

$u^{2}$

$=\frac{1}{2}\left\{(1-\gamma) \sigma+\gamma+\sqrt{(1-\gamma)^{2}\left(\sigma^{2}+\rho^{2}\right)+2 \gamma(1-\gamma) \sigma+\gamma^{2}}\right\}$

$:=\frac{1}{2} E_{\gamma}(\sigma)$.

Since $E_{\gamma}$ is increasing on the interval $\left(-\infty,-\left(1+\rho^{2}\right) / 2\right)$, for $\sigma \leq-\left(1+\rho^{2}\right) / 2$, we have

$$
\begin{aligned}
& E_{\gamma}(\sigma) \\
& \leq E_{\gamma}\left(-\left(1+\rho^{2}\right) / 2\right) \\
& =G_{\gamma}(\rho)+\sqrt{G_{\gamma}^{2}(\rho)+(1-\gamma)^{2} \rho^{2},}
\end{aligned}
$$

where

$$
G_{\gamma}(\rho)=-\frac{1}{2}(1-\gamma)\left(1+\rho^{2}\right)+\gamma .
$$

Now we define a function $F_{\gamma}: \mathbb{R} \rightarrow \mathbb{R}$ by

$$
F_{\gamma}(\rho)=G_{\gamma}(\rho)+\sqrt{G_{\gamma}^{2}(\rho)+(1-\gamma)^{2} \rho^{2}} .
$$

We note that $F_{\gamma}$ is continuous on $\mathbb{R}$ and is even. Since $F_{\gamma}^{\prime}(0)=0$ and $F_{\gamma}$ is decreasing on $(0, \infty)$ for $1 / 2 \leq \gamma<1$,

$$
F_{\gamma}(\rho) \leq F_{\gamma}(0)=3 \gamma-1
$$

for $\rho \in \mathbb{R}$. Hence

$$
u^{2} \leq \frac{1}{2} F_{\gamma}(\rho) \leq \frac{3}{2} \gamma-\frac{1}{2} .
$$

Therefore,

$$
u \leq \sqrt{\frac{3}{2} \gamma-\frac{1}{2}}=\alpha .
$$

And this shows that $\operatorname{Re}\{\psi(\mathrm{i} \rho, \sigma)\} \notin \Omega_{\alpha}$ for all $\rho$, 
$\sigma \in \mathbb{R}$ with $\sigma \leq-\left(1+\rho^{2}\right) / 2$. By Lemma 2.1, we get $\operatorname{Re}\{p(z)\}>0$ in $\mathbb{U}$ and this shows that the inequality (5) holds and the proof of Theorem 2.2 is completed.

Theorem 2.3. Let $f \in A, \beta>1$ and

$$
\operatorname{Re}\left\{\sqrt{f^{\prime}(z)}\right\}<\beta \quad(z \in \mathbb{U}) .
$$

Then

$$
\operatorname{Re}\left\{\frac{f(z)}{z}\right\}<\frac{2}{3} \beta^{2}+\frac{1}{3} \quad(z \in \mathbb{U}) .
$$

Proof. We put

$$
\delta=\frac{2}{3} \beta^{2}+\frac{1}{3}
$$

and note that $\delta>1$ for $\beta>1$. And let

$$
p(z)=\frac{1}{1-\delta}\left(\frac{f(z)}{z}-\delta\right)
$$

and

$$
\psi(r, s)=\sqrt{(1-\gamma) r+(1-\gamma) s+\gamma} .
$$

And, we put

$$
\psi(\mathrm{i} \rho, \sigma)=\sqrt{(1-\gamma) \mathrm{i} \rho+(1-\gamma) \sigma+\gamma}:=u+\mathrm{i} v,
$$

where $u$ and $v$ are real numbers. As in the proof of Theorem 2.2, we can get

$$
\left\{\psi\left(p(z), z p^{\prime}(z)\right): z \in \mathbb{U}\right\} \subset\{w \in \mathbb{C}: \operatorname{Re}\{w\}<\beta\}:=\Omega_{\beta},
$$

by (6). And

$$
\begin{aligned}
& u^{2}=\frac{1}{2}\left\{(1-\delta) \sigma+\delta+\sqrt{(1-\delta)^{2}\left(\sigma^{2}+\rho^{2}\right)+2 \delta(1-\delta) \sigma+\delta^{2}}\right\} \\
& :=\frac{1}{2} E_{\delta}(\sigma) .
\end{aligned}
$$

Since $E_{\delta}$ is decreasing on the interval $\left(-\infty,-\left(1+\rho^{2}\right) / 2\right)$, for $\sigma \leq-\left(1+\rho^{2}\right) / 2$, we have

$$
\begin{aligned}
& E_{\delta}(\sigma) \geq E_{\delta}\left(-\left(1+\rho^{2}\right) / 2\right) \\
& =G_{\delta}(\rho)+\sqrt{G_{\delta}^{2}(\rho)+(1-\delta)^{2} \rho^{2}},
\end{aligned}
$$

where

$$
G_{\delta}(\rho)=-\frac{1}{2}(1-\delta)\left(1+\rho^{2}\right)+\delta .
$$

Now we define a function $F_{\delta}: \mathbb{R} \rightarrow \mathbb{R}$ by

$$
F_{\delta}(\rho)=G_{\delta}(\rho)+\sqrt{G_{\delta}^{2}(\rho)+(1-\delta)^{2} \rho^{2}} .
$$

We note that $F_{\delta}$ is continuous on $\mathbb{R}$ and is even. Since $F_{\delta}^{\prime}(0)=0$ and $F_{\delta}$ is increasing on $(0, \infty)$ for $\delta>1$,

$$
F_{\delta}(\rho) \geq F_{\delta}(0)=3 \delta-1
$$

for $\rho \in \mathbb{R}$. Hence

$$
u^{2} \geq \frac{1}{2} F_{\delta}(\rho) \geq \frac{3}{2} \delta-\frac{1}{2} .
$$

Therefore,

$$
u \geq \sqrt{\frac{3}{2} \delta-\frac{1}{2}}=\beta .
$$

And this shows that $\operatorname{Re}\{\psi(\mathrm{i} \rho, \sigma)\} \notin \Omega_{\beta}$ for all $\rho$, $\sigma \in \mathbb{R}$ with $\sigma \leq-\left(1+\rho^{2}\right) / 2$. By Lemma 2.1, we get $\operatorname{Re}\{p(z)\}>0$ in $\mathbb{U}$ and this shows that the inequality (7) holds and the proof of Theorem 2.3 is completed.

By combining Theorem 2.2 and 2.3, we can get the following Theorem.

Theorem 2.4. Let $\alpha$ and $\beta$ be real numbers such that $1 / 2 \leq \alpha<1<\beta$ and let $f$ be a function in the class $T(\alpha, \beta)$. Then

$$
\frac{2}{3} \alpha^{2}+\frac{1}{3}<\operatorname{Re}\left\{\frac{f(z)}{z}\right\}<\frac{2}{3} \beta^{2}+\frac{1}{3} \quad(z \in \mathbb{U}) .
$$

\section{Coefficient Problems Involving Functions in $T(\alpha, \beta)$}

In the present section, we will solve some coefficient problems involving functions in the class $T(\alpha, \beta)$. And our first result on the coefficient estimates involves the function class $T(\alpha, \beta)$ and the following Lemma will be needed.

Lemma 3.1. (see Rogosinski [6]) Let

$$
q(z)=\sum_{n=1}^{\infty} B_{n} z^{n}
$$

be analytic and univalent in $\mathbb{U}$ and suppose that $q(z)$ maps $\mathbb{U}$ onto a convex domain. If

$$
p(z)=\sum_{n=1}^{\infty} A_{n} z^{n}
$$

is analytic in $\mathbb{U}$ and satisfies the following subordination:

$$
p(z) \prec q(z) \quad(z \in \mathbb{U}) .
$$

Then

$$
\left|A_{n}\right| \leq\left|B_{1}\right| \quad(n \in \mathbb{N})
$$

Theorem 3.2. Let $\alpha$ and $\beta$ be real numbers such that $0 \leq \alpha<1<\beta$. If the function

$$
f(z)=z+\sum_{n=1}^{\infty} a_{n} z^{n} \in T(\alpha, \beta),
$$

then 


$$
\left|a_{n}\right| \leq \frac{\left|B_{1}\right|}{n}\left(2+(n-2)\left|B_{1}\right|\right) \quad(n=2,3, \cdots),
$$

where $\left|B_{1}\right|$ is given by

$$
\left|B_{1}\right|=\frac{2(\beta-\alpha)}{\pi} \sin \left(\frac{1-\alpha}{\beta-\alpha} \pi\right) .
$$

Proof. Let us define

$$
q(z)=\sqrt{f^{\prime}(z)}
$$

and

$$
p(z)=1+\frac{\beta-\alpha}{\pi} \mathrm{i} \log \left(\frac{1-\mathrm{e}^{2 \pi \mathrm{i} \frac{1-\alpha}{\beta-\alpha}} \mathrm{z}}{1-\mathrm{z}}\right) .
$$

Then, the subordination (2) can be written as follows:

$$
q(z) \prec p(z) \quad(z \in \mathbb{U}) .
$$

Note that the function $p(z)$ defined by $(10)$ is convex in $\mathbb{U}$ and has the form

$$
p(z)=1+\sum_{n=1}^{\infty} B_{n} z^{n},
$$

where

$$
B_{n}=\frac{\beta-\alpha}{n \pi} \mathrm{i}\left(1-\mathrm{e}^{2 n \pi i \frac{1-\alpha}{\beta-\alpha}}\right) \quad(n \in \mathbb{N}) .
$$

If we let

$$
q(z)=1+\sum_{n=1}^{\infty} A_{n} z^{n},
$$

then by Lemma 3.1, we see that the subordination (11) implies that

$$
\left|A_{n}\right| \leq\left|B_{1}\right| \quad(n \in \mathbb{N})
$$

where

$$
\left|B_{1}\right|=\frac{2(\beta-\alpha)}{\pi} \sin \left(\frac{1-\alpha}{\beta-\alpha} \pi\right) .
$$

Now, the equality (9) implies that $f^{\prime}(z)=q^{2}(z)$. And if $n$ is even, the coefficient of $z^{n}$ in both sides lead to

$$
n a_{n}=2 A_{n-1}+2 A_{n-2} A_{1}+\cdots+2 A_{n / 2} A_{(n / 2)-1},
$$

which is the sum of $n / 2$ terms. Hence,

$$
\begin{aligned}
& n\left|a_{n}\right| \leq 2\left|A_{n-1}\right|+2\left|A_{n-2}\right|\left|A_{1}\right|+\cdots+2\left|A_{n / 2}\right|\left|A_{(n / 2)-1}\right| \\
& \leq 2\left|B_{1}\right|+2\left|B_{1}\right|^{2}+\cdots+2\left|B_{1}\right|^{2} \\
& =\left|B_{1}\right|\left(2+(n-2)\left|B_{1}\right|\right),
\end{aligned}
$$

which leads to the inequality (8). If $n$ is odd,

$$
n a_{n}=2\left(A_{n-1}+A_{n-2} A_{1}+\cdots+A_{(n+1) / 2} A_{(n-3) / 2}\right)+A_{(n-1) / 2}^{2},
$$

which is the sum of $(n-1) / 2$ terms in the bracket. Hence, we get

$$
\begin{aligned}
& n\left|a_{n}\right| \\
& \leq 2\left(\left|A_{n-1}\right|+\left|A_{n-2}\right|\left|A_{1}\right|+\cdots+\left|A_{(n+1) / 2}\right|\left|A_{(n-3) / 2}\right|\right)+\left|A_{(n-1) / 2}\right|^{2} \\
& \leq 2\left(\left|B_{1}\right|+\left|B_{1}\right|^{2}+\cdots+\left|B_{1}\right|^{2}\right)+\left|B_{1}\right|^{2} \\
& =\left|B_{1}\right|\left(2+(n-2)\left|B_{1}\right|\right),
\end{aligned}
$$

which leads to the inequality (8). Therefore, the proof of Theorem 3.2 is completed.

And now, we shall solve the Fekete-Szegö problem for $f \in T(\alpha, \beta)$ and we will need the following Lemma:

Lemma 3.3. (see Keogh and Merkers [7]) Let $p(z)=1+c_{1} z+c_{2} z^{2}+\cdots$ be a function with positive real part in $\mathbb{U}$. Then, for any complex number $v$,

$$
\left|c_{2}-v c_{1}^{2}\right| \leq 2 \max \{1 ;|1-2 v|\} .
$$

Now, the following result holds for the coefficient of $f \in T(\alpha, \beta)$.

Theorem 3.4. Let $0 \leq \alpha<1<\beta$ and let the function $f(z)$ given by $f(z)=z+\sum_{n=2}^{\infty} a_{n} z^{n}$ be in the class $T(\alpha, \beta)$. Then, for a complex number $\mu$,

$$
\begin{aligned}
& \left|a_{3}-\mu a_{2}^{2}\right| \leq \frac{4(\beta-\alpha)}{3 \pi} \sin \left(\frac{1-\alpha}{\beta-\alpha} \pi\right) \\
& \cdot \max \left\{1 ;\left|\frac{1}{2}+\lambda+\left(\frac{1}{2}-\lambda\right) \mathrm{e}^{2 \pi \mathrm{i} \frac{1-\alpha}{\beta-\alpha}}\right|\right\},
\end{aligned}
$$

where

$$
\lambda=\frac{\beta-\alpha}{2 \pi} \mathrm{i}(1-3 \mu)
$$

Proof. Let us consider a function $q(z)$ given by

$$
q(z)=\sqrt{f^{\prime}(z)} .
$$

Then, since $f \in T(\alpha, \beta)$, we have

$$
q(z) \prec p(z) \quad(z \in \mathbb{U}),
$$

where

$$
\begin{aligned}
p(z) & =1+\frac{\beta-\alpha}{\pi} \mathrm{i} \log \left(\frac{1-\mathrm{e}^{2 \pi \mathrm{i} \frac{1-\alpha}{\beta-\alpha}} \mathrm{z}}{1-\mathrm{z}}\right) \\
& =1+\sum_{n=1}^{\infty} B_{n} z^{n}
\end{aligned}
$$

with $B_{n}$ is given by (3). Let

$$
h(z)=\frac{1+p^{-1}(q(z))}{1-p^{-1}(q(z))}=1+h_{1} z+h_{2} z^{2}+\cdots .
$$


Then $h$ is analytic and has positive real part in the open unit disk $\mathbb{U}$. We also have

$$
q(z)=p\left(\frac{h(z)-1}{h(z)+1}\right) .
$$

We find from the equations (12) and (13) that

$$
a_{2}=\frac{1}{2} B_{1} h_{1}
$$

and

$$
a_{3}=\frac{1}{3} B_{1} h_{2}-\frac{1}{6} B_{1} h_{1}^{2}+\frac{1}{6} B_{2} h_{1}^{2}+\frac{1}{12} B_{1}^{2} h_{1}^{2},
$$

which imply that

$$
a_{3}-\mu a_{2}^{2}=\frac{1}{3} B_{1}\left(h_{2}-v h_{1}^{2}\right),
$$

where

$$
v=\frac{1}{2}-\frac{B_{2}}{2 B_{1}}-\frac{1}{4} B_{1}+\frac{3}{4} \mu B_{1} .
$$

Applying Lemma 3.3, we can obtain

$$
\begin{aligned}
& \left|a_{3}-\mu a_{2}^{2}\right|=\frac{1}{3}\left|B_{1}\right|\left|h_{2}-v h_{1}^{2}\right| \\
& \leq \frac{2}{3}\left|B_{1}\right| \cdot \max \{1 ;|1-2 v|\} .
\end{aligned}
$$

And substituting

$$
B_{1}=\frac{\beta-\alpha}{\pi} \mathrm{i}\left(1-\mathrm{e}^{2 \pi \mathrm{i} \frac{1-\alpha}{\beta-\alpha}}\right)
$$

and

$$
B_{2}=\frac{\beta-\alpha}{2 \pi} \mathrm{i}\left(1-\mathrm{e}^{4 \pi \mathrm{i} \frac{1-\alpha}{\beta-\alpha}}\right)
$$

in (14), we can obtain the result as asserted.

Using Theorem 3.4, we can get the following result.

Corollary 3.1. Let $0 \leq \alpha<1<\beta$ and let the function $f$, given by $f(z)=\sum_{n=2}^{\infty} a_{n} z^{n}$, be in the class $T(\alpha, \beta)$. Also let the function $f^{-1}$, defined by

$$
f^{-1}(f(z))=z=f\left(f^{-1}(z)\right)
$$

be the inverse of $f$. If

$$
f^{-1}(w)=w+\sum_{n=2}^{\infty} b_{n} w^{n} \quad\left(|w|<r_{0} ; r_{0}>\frac{1}{4}\right),
$$

then

$$
\left|b_{2}\right| \leq \frac{2(\beta-\alpha)}{\pi} \sin \left(\frac{1-\alpha}{\beta-\alpha} \pi\right)
$$

and

$$
\begin{aligned}
& \left|b_{3}\right| \leq \frac{4(\beta-\alpha)}{3 \pi} \sin \left(\frac{1-\alpha}{\beta-\alpha} \pi\right) \\
& \cdot \max \left\{1 ;\left|\frac{1}{2}-\phi+\left(\frac{1}{2}+\phi\right) \mathrm{e}^{2 \pi \mathrm{i} \frac{1-\alpha}{\beta-\alpha}}\right|\right\},
\end{aligned}
$$

where

$$
\phi=\frac{5}{2 \pi}(\beta-\alpha) \mathrm{i} .
$$

Proof. The relations (17) and (18) give

$$
b_{2}=-a_{2}
$$

and

$$
b_{3}=2 a_{2}^{2}-a_{3} .
$$

Thus, we can get the estimate for $\left|b_{2}\right|$ by

$$
\left|b_{2}\right|=\left|a_{2}\right| \leq\left|B_{1}\right|=\frac{2(\beta-\alpha)}{\pi} \sin \left(\frac{1-\alpha}{\beta-\alpha} \pi\right),
$$

immediately. Furthermore, an application of Theorem 3.4 (with $\mu=2$ ) gives the estimates for $\left|b_{3}\right|$, hence the proof of Corollary 3.1 is completed.

Finally, we shall estimate on some initial coefficients for the bi-univalent functions $f \in T_{\sigma}(\alpha, \beta)$.

Theorem 3.5. For given $\alpha$ and $\beta$ such that $0 \leq \alpha<1<\beta$, let $f$ be given by $f(z)=z+\sum_{n=2}^{\infty} a_{n} z^{n}$ be in the class $T_{\sigma}(\alpha, \beta)$. Then

$$
\left|a_{2}\right| \leq \sqrt{\frac{2(\beta-\alpha)}{\pi} \sin (\varphi)(1+\sin (\varphi))}
$$

and

$$
\left|a_{3}\right| \leq \frac{2(\beta-\alpha)}{\pi} \sin (\varphi)\left(1+\frac{7}{3} \sin (\varphi)\right)
$$

with $\varphi=\frac{1-\alpha}{\beta-\alpha} \pi$.

Proof. If $f \in T_{\sigma}(\alpha, \beta)$, then $f \in T(\alpha, \beta)$ and $g \in T(\alpha, \beta)$, where

$$
g(z)=f^{-1}(z)=z+\sum_{n=2}^{\infty} b_{n} z^{n} .
$$

Hence

$$
Q(z):=\sqrt{f^{\prime}(z)} \prec p(z) \quad(z \in \mathbb{U})
$$

and

$$
L(z):=\sqrt{g^{\prime}(z)} \prec p(z) \quad(z \in \mathbb{U}),
$$

where $p(z)$ is given by (1). Let

$$
h(z)=\frac{1+p^{-1}(Q(z))}{1-p^{-1}(Q(z))}=1+h_{1} z+h_{2} z^{2}+\cdots
$$


and

$$
k(z)=\frac{1+p^{-1}(L(z))}{1-p^{-1}(L(z))}=1+k_{1} z+k_{2} z^{2}+\cdots .
$$

Then $h$ and $k$ are analytic and have positive real part in $\mathbb{U}$. Also, we have

$$
Q(z)=p\left(\frac{h(z)-1}{h(z)+1}\right)
$$

and

$$
L(z)=p\left(\frac{k(z)-1}{k(z)+1}\right)
$$

By suitably comparing coefficient, we get

$$
\begin{gathered}
a_{2}=\frac{1}{2} B_{1} h_{1} \\
a_{3}=\frac{1}{3} B_{1} h_{2}-\frac{1}{6} B_{1} h_{1}^{2}+\frac{1}{6} B_{2} h_{1}^{2}+\frac{1}{12} B_{1}^{2} h_{1}^{2} \\
b_{2}=\frac{1}{2} B_{1} k_{1}
\end{gathered}
$$

and

$$
b_{3}=\frac{1}{3} B_{1} k_{2}-\frac{1}{6} B_{1} k_{1}^{2}+\frac{1}{6} B_{2} k_{1}^{2}+\frac{1}{12} B_{1}^{2} k_{1}^{2},
$$

where $B_{1}$ and $B_{2}$ are given by (15) and (16), respectively. Now, considering (21) and (23), we get

$$
h_{1}=-k_{1} \text {. }
$$

Also, from (22),(23),(24) and (25), we find that

$$
4 a_{2}^{2}=B_{1}\left(h_{2}+k_{2}\right)+h_{1}^{2}\left(B_{2}-B_{1}\right) .
$$

Therefore, we have

$$
\begin{aligned}
& 4\left|a_{2}\right|^{2} \leq\left|B_{1}\right|\left(\left|h_{2}\right|+\left|k_{2}\right|\right)+\left|h_{1}\right|^{2}\left|B_{2}-B_{1}\right| \\
& \leq 4\left|B_{1}\right|+4\left|B_{2}-B_{1}\right| .
\end{aligned}
$$

This gives the bound on $\left|a_{2}\right|$ as asserted in (19). Now, further computations from (22), (24)-(26) lead to

$$
a_{3}=\frac{1}{12} B_{1}\left(5 h_{2}+k_{2}\right)+\frac{7}{12} h_{1}^{2}\left(B_{2}-B_{1}\right) .
$$

This equation, together with the well-known estimates [8]:

$$
\left|h_{1}\right| \leq 2,\left|h_{2}\right| \leq 2 \text { and }\left|k_{2}\right| \leq 2
$$

lead us to the inequality (20). Therefore, the proof of Theorem 3.5 is completed.

\section{Acknowledgements}

The research was supported by Kyungsung University Re-search Grants in 2013.

\section{REFERENCES}

[1] K. Kuroki and S. Owa, "Notes on New Class for Certain Analytic Functions," RIMS Kokyuroku 1772, 2011, pp. 21-25.

[2] H. M. Srivastava, A. K. Mishra and P. Gochhayat, "Certain Subclasses of Analytic and Bi-Univalent Functions," Applied Mathematics Letters, Vol. 23, No. 10, 2010, pp. 1188-1192. doi:10.1016/j.aml.2010.05.009

[3] Q.-H. Xu, Y.-C. Gui and H. M. Srivastava, "Coefficient Estimates for a Certain Subclass of Analytic and Bi-Univalent Functions," Applied Mathematics Letters, Vol. 25, No. 6, 2012, pp. 990-994. doi:10.1016/i.aml.2011.11.013

[4] R. M. Ali, K. Lee, V. Ravichandran and S. Supramaniam, "Coefficient Estimates for Bi-Univalent Ma-Minda Starlike and Convex Functions," Applied Mathematics Letters, Vol. 25, No. 3, 2012, pp. 344-351.

[5] S. S. Miller and P. T. Mocanu, "Differential Subordinations, Theory and Applications," Marcel Dekker, 2000.

[6] W. Rogosinski, "On the Coefficients of Subordinate Functions," Proceeding of the London Mathematical Society, Vol. 2, No. 48, 1943, pp. 48-62.

[7] F. Keogh and E. Merkers, "A Coefficient Inequality for Certain Classes of Analytic Functions," Proceedings of the American Mathematical Society, Vol. 20, No. 1, 1969, pp. 8-12. doi:10.1090/S0002-9939-1969-0232926-9

[8] P. Duren, "Univalent Functions," Springer-Verlag, New York, 1983. 